\title{
Locally and Globally Exponential Synchronization of Moving Agent Networks by Adaptive Control
}

\author{
Lifu Wang, ${ }^{1}$ Peng Xue, ${ }^{2}$ Zhi Kong, and Xingang Wang ${ }^{1}$ \\ ${ }^{1}$ School of Control Engineering, Northeastern University at Qinhuangdao, Qinhuangdao 066004, China \\ ${ }^{2}$ Department of Electrical Information Engineering, Henan Institute of Engineering, Zhengzhou 451191, China \\ Correspondence should be addressed to Lifu Wang; wlfkz@qq.com
}

Received 18 February 2013; Accepted 23 September 2013

Academic Editor: Yannick De Decker

Copyright (C) 2013 Lifu Wang et al. This is an open access article distributed under the Creative Commons Attribution License, which permits unrestricted use, distribution, and reproduction in any medium, provided the original work is properly cited.

\begin{abstract}
The exponential synchronization problem is investigated for a class of moving agent networks in a two-dimensional space and exhibits time-varying topology structure. Based on the Lyapunov stability theory, adaptive feedback controllers are developed to guarantee the exponential synchronization between each agent node. New criteria are proposed for verifying the locally and globally exponential synchronization of moving agent networks under the constraint of fast switching. In addition, a numerical example, including typical moving agent network with the Rössler system at each agent node, is provided to demonstrate the effectiveness and applicability of the proposed design approach.
\end{abstract}

\section{Introduction}

Over the past decade years, the analysis of complex systems from the viewpoint of networks has become an important interdisciplinary issue [1]. Complex networks have been intensively studied in many fields, such as social, biological, mathematical, and engineering sciences. Generally, a complex network is made up of interconnected nodes in which a node is a basic unit with detailed contents. These interactions between nodes determine many basic properties of a network. To better understand the complex dynamical behaviors of many natural systems, we need to study their operating mechanism, dynamic behavior, synchronization, antijamming ability, and so on. Recently, synchronization of complex dynamical networks has received a great deal of attentions from various fields of science and engineering [1-3]. Particularly, synchronization of large-scale complex networks of coupled chaotic oscillators has been extensively investigated in the fields of science and engineering.

The synchronization properties of a complex network are mainly determined by its topological structures connections between nodes. In the current study of complex networks, most of the existing works on synchronization consider static networks, whose topological structures do not change as time evolves [4-10]. The Master-stability function (MSF) approach [11] allows us to determine the stability of a linearly coupled dynamical network with a constant coupling (or Laplacian) matrix. However, numerous real-world networks such as biological, communication, social, and epidemiological networks generally evolve with time-varying topological structures. Henceforth, researchers have devoted more and more efforts to complex networks with time-varying topologies. Stilwell et al. [12] prove that if the network of oscillators synchronizes for the static time-average of the topology, then the network will synchronize with the time-varying topology if the time-average is achieved sufficiently fast. At the same conditions, Lu et al. $[13,14]$ found that the directed network with switching topology can reach global synchronization for sufficiently large coupling strength if there exists a spanningdirected tree in the network.

Inspired by the above discussions, in this paper we investigate the adaptive exponential synchronization problem for a specific time-varying network model. The model arises from the interaction of mobile agents proposed by Frasca et al. [15] and can be widely used to explore various practical problems, for example, clock synchronization in mobile robots [16], synchronized bulk oscillations [17], and task coordination of swarming animals [18]. How one controls the appearance 
of synchronized states of the dynamical network is of great significance in theory and potential applications. For the mobile agent network model, we introduce adaptive control to regulate its synchronization, as an attempt to explain the control of complex time-varying systems.

Although synchronization control of moving agent network has great application potential in a variety of areas, there has been very little existing literature on the exponential synchronization problem. Therefore, we adopt the constraint of fast switching to derive exponent synchronization conditions. By using Lyapunov stability theory, adaptive controllers are designed for synchronization of moving agent network with time-varying topological structures. The adaptive controllers can ensure the states of moving agent network fast synchronization.

The current paper is organized as follows. A general moving agent network model and several mathematical preliminaries are introduced in Section 2. In Section 3, several locally and globally adaptive synchronization criteria for the moving agent networks are deduced. A representative example is given to show the effectiveness of the proposed network synchronization criteria in Section 4. Conclusions are finally drawn in Section 5.

\section{Moving Agent Network Model and Preliminaries}

We consider $N$ as moving agents distributed in a twodimensional planar space of size $L \Gamma=\left\{\left(y_{1}, y_{2}\right) \in R^{2}: 0 \leq\right.$ $\left.y_{1} \leq L, 0 \leq y_{2} \leq L\right\}$, with periodic boundary conditions. Each agent moves with velocity $v_{i}(t)$ and direction of motion $\theta_{i}(t)$. The velocity $v_{i}(t)$ is the same for all individuals (denoted by $v$ ) and is updated in direction through the angle $\theta_{i}(t)$ for each time unit. The agents are considered as random walkers. Hence, the motion law of the ith agent is given as follows:

$$
\begin{gathered}
y_{i 1}\left(t+\Delta t_{M}\right)=y_{i 1}(t)+v \cos \theta_{i}(t) \Delta t_{M}, \\
y_{i 2}\left(t+\Delta t_{M}\right)=y_{i 2}(t)+v \sin \theta_{i}(t) \Delta t_{M}, \\
\theta_{i}\left(t+\Delta t_{M}\right)=\eta_{i}\left(t+\Delta t_{M}\right),
\end{gathered}
$$

where $i=1,2, \ldots, N, y_{i}(t)=\left(y_{1}, y_{2}\right) \in \Gamma$ is the position of agent $i$ in the plane at time $t, \eta_{i}(t)$ are $N$ independent random variables chosen at each time unit with uniform probability in the interval $[0,2 \pi]$, and $\Delta t_{M}$ is the motion integration step size.

Each agent interacts at a given time with only those agents located within a neighborhood of an interaction radius, defined as $R[15,19,20]$. When two agents interact, the state equations of each agent are changed to include diffusive coupling with the neighboring agent. Under these hypotheses, the state dynamics of agent node $i$ can be formulated as

$$
\dot{x}_{i}(t)=f\left(x_{i}(t), t\right)-\sigma \sum_{j=1}^{N} g_{i j}(t) x_{j}(t)+u_{i}(t),
$$

where $i=1, \ldots, N, x_{i}(t)=\left(x_{i 1}(t), x_{i 2}(t), \ldots, x_{i n}(t)\right)^{T} \in R^{n}$ are the state variables of the node $i$; $: D \times R^{+} \rightarrow R^{n}$ is a smooth nonlinear vector-valued function which governs the local dynamics of oscillator; $\sigma$ is the coupling strength; $g_{i j}(t)$ are the elements of a time-varying Laplacian matrix: $G(t)=$ $\left[g_{i j}(t)\right] \in R^{N \times N}$ which defines the neighborhood of agents at a given time $t$ and depends on the trajectory of each agent. In detail, for arbitrary two agents $i, j$ with distance $d$ at time $t$, $g_{i j}(t)=-1, g_{j i}(t)=-1$ if $d<R ; g_{i j}(t)=0, g_{j i}(t)=0$ if $d>R$; $g_{i i}(t)=h$, where $h$ is the number of neighbors of the $i$ th agent at time $t ; u_{i}(t) \in R^{n}$ are the control inputs.

In this paper, the control objective is to make the states of network (2) exponentially synchronize with a manifold defined in (3) by introducing a simple adaptive controller into each individual node

$$
x_{1}(t)=x_{2}(t)=\cdots=x_{N}(t)=s(t),
$$

where $s(t)$ is a solution of an isolated node,

$$
\dot{s}(t)=f(s(t), t) .
$$

We assume that $s(t)$ is an arbitrary desired state which can be an equilibrium point, a periodic orbit, an aperiodic orbit, or even a chaotic orbit in the phase space.

Next, the rigorous mathematical definition of exponential synchronization for dynamical network (2) is introduced.

Definition 1. Let $X\left(t, t_{0}, \phi\right)=\left(x_{1}\left(t, t_{0}, \phi\right)^{T}, x_{2}\left(t, t_{0}, \phi\right)^{T}, \ldots\right.$, $\left.x_{N}\left(t, t_{0}, \phi\right)^{T}\right)^{T} \in R^{n N}$ be a solution of dynamical network (2), where $\phi=\left(\phi_{1}^{T}, \phi_{2}^{T}, \ldots, \phi_{N}^{T}\right)^{T}$ are initial conditions of node $i$ and $f: D \times R^{+} \rightarrow R^{n}$ is continuously differentiable on $D \subseteq$ $R^{n}$. If there are control inputs $u_{i}, i=1,2, \ldots, N$, and further there exist constants $\alpha>0, \lambda>0$ and a nonempty subset $\Lambda \subseteq D$ with $\phi_{i} \in \Lambda, i=1,2, \ldots, N$, such that $X\left(t, t_{0}, \phi\right) \in$ $D \times D \times \cdots \times D$ for all $t \geq t_{0}$, and

$$
\left\|X\left(t, t_{0}, \phi\right)-s\left(t, t_{0}, s_{0}\right)\right\| \leq \alpha e^{-\lambda t}\left\|\phi-S_{0}\right\|
$$

(Hereafter, denote $\|\cdot\|$ as the Euclidean norm.) where $S\left(t, t_{0}, s_{0}\right)=\left(s\left(t, t_{0}, s_{0}\right)^{T}, \ldots, s\left(t, t_{0}, s_{0}\right)^{T}\right)^{T} \in R^{n N}, S_{0}=\left(s_{0}^{T}\right.$, $\left.\ldots, s_{0}^{T}\right)^{T}, s\left(t, t_{0}, s_{0}\right)$ is a solution of the system (4) with the initial condition $s_{0} \in D$, then the dynamical network (2) is said to realize exponential synchronization such that $\lambda$ is the exponential rate. And $\Lambda \times \cdots \times \Lambda$ is called the region of synchrony for the dynamical network (2) (see [21, 22]).

\section{Synchronization of Moving Agent Networks}

In this section, we discuss the exponential synchronization of moving agent network (2) by designing adaptive controllers for each agent node. Several network synchronization criteria are given.

3.1. Local Synchronization. In order to achieve the objective of synchronization on the manifold (3), let us define the error vector

$$
e_{i}(t)=x_{i}(t)-s(t), \quad i=1,2, \ldots, N
$$


Subtracting (4) from (2) yields the error dynamical system

$$
\dot{e}_{i}(t)=f\left(x_{i}(t), t\right)-f(s(t), t)-\sigma \sum_{j=1}^{N} g_{i j}(t) e_{j}(t)+u_{i}(t) .
$$

Then, exponential synchronization problem of the dynamical network (2) is equivalent to the problem of exponential stabilization of the error dynamical system (7).

Linearizing error system (7) about the synchronized states $s(t)$, we can get

$$
\dot{e}_{i}(t)=A(t) e_{i}(t)-\sigma \sum_{j=1}^{N} g_{i j}(t) e_{j}(t)+u_{i}(t),
$$

where $i=1,2, \ldots, N$ and $A(t)=J_{f}(s, t)$ is the Jacobian matrix of $f$ evaluated at $x=s(t)$.

In the following, we give several useful hypotheses.

Assumption 2. Suppose that there exists a nonnegative constant $\alpha$ satisfying

$$
\|A(t)\| \leq \beta .
$$

Generally, Assumption 2 is likely to be satisfied. For example, in many chaotic systems such as Lorenz system, Rössler system, and Chen system, there exists a constant $\beta$ satisfying $\|A(t)\| \leq \beta$.

Assumption 3. Suppose there exists a constant $T$ such that coupling matrix $G(t)$ satisfying

$$
\frac{1}{T} \int_{t}^{t+T} G(\tau) d \tau=\bar{G},
$$

where $\bar{G}$ is the time-average of the coupling matrix $G(t)$.

Assumption 3 implies that the switching between all the possible network configurations is sufficiently fast as defined in [12]. According to [12] the following lemma can be given.

Lemma 4. Suppose that a coupled network with fixed topology defined by

$$
\dot{x}_{i}(t)=f\left(x_{i}(t), t\right)-\sigma \sum_{j=1}^{N} \bar{g}_{i j} x_{j}(t)+u_{i}(t)\left(\bar{G}=\left[\bar{g}_{i j}\right]\right)
$$

admits a stable synchronization manifold and Assumption 3 holds. Then the network with a time-variant topological structure defined by (2) admits a stable synchronization manifold.

The proof of Lemma 4 can be obtained by main results of [12]. Here we omitted proof process.

According to analysis of [15], under the constraint of fast switching, $\bar{G}=p G_{A}$, where $p$ is the probability that a link is activated and thus $p=\pi R^{2} / L^{2}$, and $G_{A}$ is the all-to-all coupling matrix with zero-row sum. That is

$$
\bar{G}=\frac{\pi R^{2}}{L^{2}}\left[\begin{array}{cccc}
N-1 & -1 & \cdots & -1 \\
-1 & N-1 & \cdots & -1 \\
\vdots & \vdots & \ddots & \vdots \\
-1 & -1 & \cdots & N-1
\end{array}\right] .
$$

Based on Assumptions 2 and 3, a network synchronization criterion is deduced as follows.

Theorem 5. Suppose that Assumptions 2 and 3 hold. Then the dynamical moving agent network (2) is locally exponential synchronization under the following sets of adaptive controllers:

$$
u_{i}=-d_{i} e_{i}, \quad i=1,2, \ldots, N
$$

and updating laws

$$
\dot{d}_{i}=e^{2 \lambda t}\left\|e_{i}\right\|^{2},
$$

where $\lambda$ is the exponential rate available to be designed, and $d_{i}\left(t_{0}\right)=\alpha-\lambda$, where $\alpha$ is defined in the Assumption 2 .

Proof. Select a Lyapunov function as follows:

$$
V(t)=\frac{1}{2} \sum_{i=1}^{N} e_{i}^{T} e_{i}+\frac{1}{2} \sum_{i=1}^{N} e^{-2 \lambda t}\left(d_{i}-d_{i}^{*}\right)^{2}
$$

where constant $d_{i}^{*}$ is to be given below. Then the time derivative of $V(t)$ along the solution of the error system (8) is given as follows:

$$
\begin{aligned}
\dot{V}= & \frac{1}{2} \sum_{i=1}^{N}\left(\dot{e}_{i}^{T} e_{i}+e_{i}^{T} \dot{e}_{i}\right)+\frac{1}{2} \sum_{i=1}^{N} e^{-2 \lambda t}(-2 \lambda)\left(d_{i}-d_{i}^{*}\right)^{2} \\
& +\sum_{i=1}^{N} e^{-2 \lambda t}\left(d_{i}-d_{i}^{*}\right) \dot{d}_{i} \\
= & \frac{1}{2} \sum_{i=1}^{N}\left(\left(A(t) e_{i}-\sigma \sum_{j=1}^{N} g_{i j}(t) e_{j}+u_{i}\right)^{T} e_{i}\right. \\
& \left.+e_{i}^{T}\left(A(t) e_{i}-\sigma \sum_{j=1}^{N} g_{i j}(t) e_{j}+u_{i}\right)\right) \\
& -\lambda \sum_{i=1}^{N} e^{-2 \lambda t}\left(d_{i}-d_{i}^{*}\right)^{2}+\sum_{i=1}^{N} e^{-2 \lambda t}\left(d_{i}-d_{i}^{*}\right) e^{2 \lambda t} e_{i}^{T} e_{i} \\
= & \sum_{i=1}^{N} e_{i}^{T}\left(\frac{\left(A^{T}(t)+A(t)\right)}{2} e_{i}-\sigma \sum_{j=1}^{N} g_{i j}(t) e_{j}(t)+u_{i}\right) \\
& -\lambda \sum_{i=1}^{N} e^{-2 \lambda t}\left(d_{i}-d_{i}^{*}\right)^{2}+\sum_{i=1}^{N}\left(d_{i}-d_{i}^{*}\right) e_{i}^{T} e_{i} .
\end{aligned}
$$


According to Assumption 2, $\|A(t)\| \leq \beta$, so $\|\left(A^{T}(t)+A(t)\right) /$ $2 \| \leq \beta$

$$
\begin{aligned}
\dot{V} \leq & \beta \sum_{i=1}^{N} e_{i}^{T} e_{i}-\sigma \sum_{i=1}^{N} \sum_{j=1}^{N} g_{i j}(t) e_{i}^{T} e_{j}(t)-\sum_{i=1}^{N} d_{i} e_{i}^{T} e_{i} \\
& +\sum_{i=1}^{N}\left(d_{i}-d_{i}^{*}\right) e_{i}^{T} e_{i}-\lambda \sum_{i=1}^{N} e^{-2 \lambda t}\left(d_{i}-d_{i}^{*}\right)^{2} \\
= & \beta \sum_{i=1}^{N} e_{i}^{T} e_{i}-\sigma \sum_{i=1}^{N} \sum_{j=1}^{N} g_{i j}(t) e_{i}^{T} e_{j}(t) \\
& -\sum_{i=1}^{N} d_{i}^{*} e_{i}^{T} e_{i}-\lambda \sum_{i=1}^{N} e^{-2 \lambda t}\left(d_{i}-d_{i}^{*}\right)^{2} .
\end{aligned}
$$

Here, according to Lemma 4, under the constraint of fast switching, one substitute $g_{i j}(t)$ for $\bar{g}_{i j}$

$$
\begin{aligned}
\dot{V}(t) \leq & \beta \sum_{i=1}^{N} e_{i}^{T} e_{i}-\sigma \sum_{i=1}^{N} \sum_{j=1}^{N} \bar{g}_{i j} e_{i}^{T} e_{j} \\
& -\sum_{i=1}^{N} d_{i}^{*} e_{i}^{T} e_{i}-\lambda \sum_{i=1}^{N} e^{-2 \lambda t}\left(d_{i}-d_{i}^{*}\right)^{2} .
\end{aligned}
$$

Since $e_{i}^{T} e_{j} \leq(1 / 2)\left(e_{i}^{T} e_{i}+e_{j}^{T} e_{j}\right)$,

$$
\begin{aligned}
-\sigma \sum_{i=1}^{N} \sum_{j=1}^{N} \bar{g}_{i j} e_{i}^{T} e_{j} \\
=\frac{\sigma \pi R^{2}}{L^{2}}\left[-(N-1) e_{1}^{T} e_{1}+e_{1}^{T} e_{2}\right. \\
+e_{1}^{T} e_{3}+\cdots+e_{1}^{T} e_{N}+e_{2}^{T} e_{1}-(N-1) e_{2}^{T} e_{2} \\
+e_{2}^{T} e_{3}+\cdots-e_{2}^{T} e_{N} \cdots+e_{N}^{T} e_{1}+e_{N}^{T} e_{2} \\
\left.-\cdots+e_{N}^{T} e_{N-1}-(N-1) e_{N}^{T} e_{N}\right] \\
\leq \frac{\sigma \pi R^{2}}{L^{2}}\left[-(N-1) e_{1}^{T} e_{1}+\frac{1}{2}\left(e_{1}^{T} e_{1}+e_{2}^{T} e_{2}\right)\right. \\
+\frac{1}{2}\left(e_{1}^{T} e_{1}+e_{3}^{T} e_{3}\right) \\
+\cdots+\frac{1}{2}\left(e_{1}^{T} e_{1}+e_{N}^{T} e_{N}\right) \\
+\frac{1}{2}\left(e_{2}^{T} e_{2}+e_{1}^{T} e_{1}\right)-(N-1) e_{2}^{T} e_{2} \\
+ \\
+\frac{1}{2}\left(e_{2}^{T} e_{2}+e_{3}^{T} e_{3}\right) \\
+\cdots+\frac{1}{2}\left(e_{2}^{T} e_{2}+e_{N}^{T} e_{N}\right) \cdots
\end{aligned}
$$

$$
\begin{aligned}
& +\frac{1}{2}\left(e_{N}^{T} e_{N}+e_{1}^{T} e_{1}\right) \\
& +\frac{1}{2}\left(e_{N}^{T} e_{N}+e_{2}^{T} e_{2}\right) \\
& +\cdots+\frac{1}{2}\left(e_{N}^{T} e_{N}+e_{N-1}^{T} e_{N-1}\right) \\
& \left.-(N-1) e_{N}^{T} e_{N}\right]
\end{aligned}
$$

$=0$.

Therefore,

$$
\begin{aligned}
\dot{V}(t) \leq & \beta \sum_{i=1}^{N} e_{i}^{T} e_{i}-\sum_{i=1}^{N} d_{i}^{*} e_{i}^{T} e_{i} \\
& -\lambda \sum_{i=1}^{N} e^{-2 \lambda t}\left(d_{i}-d_{i}^{*}\right)^{2} .
\end{aligned}
$$

Since $\beta$ is a nonnegative constant, one can select suitable constants $d_{i}^{*}$ to make $\beta-d_{i}^{*}=\lambda$. Therefore,

$$
\begin{aligned}
\dot{V} & \leq-\lambda \sum_{i=1}^{N} e_{i}^{T} e_{i}-\lambda \sum_{i=1}^{N} e^{-2 \lambda t}\left(d_{i}-d_{i}^{*}\right)^{2} \\
& =-2 \lambda V(t) .
\end{aligned}
$$

By calculating integration on both sides of the above inequality, we get $V(t) \leq e^{-2 \lambda\left(t-t_{0}\right)} V\left(t_{0}\right)$.

According to (15), one can get $(1 / 2) \sum_{i=1}^{N} e_{i}^{T} e_{i} \leq V(t)$, so $(1 / 2) \sum_{i=1}^{N} e_{i}^{T} e_{i} \leq e^{-2 \lambda\left(t-t_{0}\right)} V\left(t_{0}\right)$

$$
V\left(t_{0}\right)=\frac{1}{2} \sum_{i=1}^{N} e_{i}^{T}\left(t_{0}\right) e_{i}\left(t_{0}\right)+\frac{1}{2} \sum_{i=1}^{N} e^{-2 \lambda t_{0}}\left(d_{i}\left(t_{0}\right)-d_{i}^{*}\right)^{2} .
$$

Let $d_{i}\left(t_{0}\right)=d_{i}^{*}$, so $(1 / 2) \sum_{i=1}^{N} e_{i}^{T} e_{i} \leq e^{-2 \lambda\left(t-t_{0}\right)}(1 /$ 2) $\sum_{i=1}^{N} e_{i}^{T}\left(t_{0}\right) e_{i}\left(t_{0}\right)$. That is,

$$
\|e(t)\|^{2} \leq e^{-2 \lambda\left(t-t_{0}\right)}\left\|e\left(t_{0}\right)\right\|^{2} .
$$

So, one gets

$$
\|e(t)\| \leq \alpha e^{-\lambda t},
$$

where $\alpha=e^{\lambda t_{0}}\left\|e\left(t_{0}\right)\right\|$.

Therefore, in closed-loop under the controllers (13) and updating laws (14), it follows that the error system (7) is locally exponentially stable at the equilibrium set $e_{i}=0$, $i=1,2, \ldots, N$, with the exponential rate $\lambda$. Consequently, the synchronous solution $S(t)$ of the dynamical network (2) is locally exponentially stable. Then the dynamical network (2) is said to realize locally exponential synchronization under the controllers (13) and updating laws (14). The proof is thus completed.

Remark 6. Since error dynamical system (8) is linearized, the moving agent network (2) is locally exponential synchronization rather than globally exponential synchronization. 
3.2. Global Synchronization. Rewrite node dynamics $\dot{x}_{i}(t)=$ $f\left(x_{i}(t), t\right)$ as $\dot{x}_{i}(t)=B x_{i}(t)+h\left(x_{i}(t), t\right)$, where $B$ is a constant matrix and $h: D \times R^{+} \rightarrow R^{n}$ is a smooth nonlinear function. Thus, network (2) is described by

$$
\dot{x}_{i}(t)=B x_{i}(t)+h\left(x_{i}(t), t\right)-\sigma \sum_{j=1}^{N} g_{i j}(t) x_{j}+u_{i}(t),
$$

where $i=1,2, \ldots, N$. Similarly, one can get the error system

$$
\dot{e}_{i}(t)=B e_{i}(t)+\bar{h}\left(x_{i}, s, t\right)-\sigma \sum_{j=1}^{N} g_{i j}(t) e_{j}(t)+u_{i}(t),
$$

where $i=1,2, \ldots, N$ and $\bar{h}\left(x_{i}, s, t\right)=h\left(x_{i}, t\right)-h(s, t)$.

Assumption 7. Suppose that there exists a nonnegative constant $\mu$, satisfying

$$
\left\|\bar{h}\left(x_{i}, s, t\right)\right\| \leq \mu\left\|e_{i}\right\| .
$$

Theorem 8. Suppose that Assumptions 3 and 7 hold. Then the dynamical moving agent network (2) is globally synchronized under the following adaptive controllers:

$$
u_{i}=-d_{i} e_{i}, \quad i=1,2, \ldots, N
$$

and updating laws

$$
\dot{d}_{i}=e^{2 \lambda t}\left\|e_{i}\right\|^{2},
$$

where $d_{i}\left(t_{0}\right)=\gamma+\mu-\lambda$, $\gamma$ is a nonnegative constant satisfying $\left\|\left(B+B^{T}\right) / 2\right\| \leq \gamma, \mu$ is defined in the Assumption 7 , and $\lambda$ is the exponential rate available to be designed.

Proof. since $B$ is a given constant matrix, there exists a nonnegative constant $\gamma$ such that $\left\|\left(B+B^{T}\right) / 2\right\| \leq \gamma$.

Similarly, construct Lyapunov function (15); then one has

$$
\begin{aligned}
\dot{V}= & \sum_{i=1}^{N} e_{i}^{T} \frac{\left(B^{T}+B\right)}{2} e_{i}+\sum_{i=1}^{N} e_{i}^{T} \bar{h}\left(x_{i}, s, t\right) \\
& -\sigma \sum_{i=1}^{N} \sum_{j=1}^{N} e_{i}^{T} g_{i j}(t) e_{j}+\sum_{i=1}^{N} e_{i}^{T} u_{i}(t) \\
& -\lambda \sum_{i=1}^{N} e^{-2 \lambda t}\left(d_{i}-d_{i}^{*}\right)^{2}+\sum_{i=1}^{N}\left(d_{i}-d_{i}^{*}\right) e_{i}^{T} e_{i} .
\end{aligned}
$$

And according to Assumption 7, there exists a nonnegative constant $\mu$, satisfying $\left\|\bar{h}\left(x_{i}, s, t\right)\right\| \leq \mu\left\|e_{i}\right\|$; therefore,

$$
\begin{aligned}
\dot{V} \leq & \left(\gamma+\mu+d_{i}-d_{i}^{*}\right) \sum_{i=1}^{N} e_{i}^{T} e_{i} \\
& -\sigma \sum_{i=1}^{N} \sum_{j=1}^{N} e_{i}^{T} g_{i j}(t) e_{j}+\sum_{i=1}^{N} e_{i}^{T} u_{i}(t) \\
& -\lambda \sum_{i=1}^{N} e^{-2 \lambda t}\left(d_{i}-d_{i}^{*}\right)^{2} .
\end{aligned}
$$

According to Lemma 4, one substitute $g_{i j}(t)$ for $\bar{g}_{i j}$

$$
\dot{V} \leq\left(\gamma+\mu-d_{i}^{*}\right) \sum_{i=1}^{N} e_{i}^{T} e_{i}-\lambda \sum_{i=1}^{N} e^{-2 \lambda t}\left(d_{i}-d_{i}^{*}\right)^{2} .
$$

Since $\gamma, \mu$ are nonnegative constants, one can select suitable constants $d_{i}^{*}$ to make $\gamma+\mu-d_{i}^{*}=\lambda$. Therefore,

$$
\dot{V} \leq-\lambda \sum_{i=1}^{N} e_{i}^{T} e_{i}-\lambda \sum_{i=1}^{N} e^{-2 \lambda t}\left(d_{i}-d_{i}^{*}\right)^{2}=-2 \lambda V(t) .
$$

By similar calculation, one gets $V(t) \leq e^{-2 \lambda\left(t-t_{0}\right)} V\left(t_{0}\right)$. Then, one has

$$
\|e(t)\|^{2} \leq e^{-2 \lambda\left(t-t_{0}\right)}\left\|e\left(t_{0}\right)\right\|^{2} .
$$

Similar, one can get

$$
\|e(t)\| \leq \alpha e^{-\lambda t}
$$

where $\alpha=e^{\lambda t_{0}}\left\|e\left(t_{0}\right)\right\|$

Therefore, in closed-loop under the controllers (28) and updating laws (29), it follows that the error system (7) is globally exponentially stable at the equilibrium set $e_{i}=0$, $i=1,2, \ldots, N$, with the globally exponential rate $\lambda$. Consequently, the synchronous solution $S(t)$ of the dynamical network (2) is globally exponential stable. Then the dynamical network (2) is said to realize globally exponential synchronization under the controllers (28) and updating laws (29). The proof is thus completed.

Remark 9. In this paper, the coupling scheme of dynamical network (2) is a linear relationship. If the network coupling scheme is general nonlinear relationship, the network (2) is rewritten as follows:

$$
\dot{x}_{i}=f\left(x_{i}\right)-\sigma \sum_{j=1}^{N} g_{i j}(t) h\left(x_{j}\right)+u_{i} .
$$

Suppose that $\left\|h\left(x_{j}\right)\right\|<\eta$ ( $\eta$ is a nonnegative constant) hold. Then, the synchronous solution $S(t)$ of moving agent network (36) is globally exponentially stable under the adaptive controllers (28) and updated laws (29) by the similar proof.

\section{Simulation}

In this section, one example is given for illustrating the proposed synchronization criteria. Consider a dynamical network consisting of 5 identical Rössler oscillators, where state dynamics of each agent is described by

$$
\left(\begin{array}{c}
\dot{x}_{i 1} \\
\dot{x}_{i 2} \\
\dot{x}_{i 3}
\end{array}\right)=B\left(\begin{array}{c}
x_{i 1} \\
x_{i 2} \\
x_{i 3}
\end{array}\right)+\left(\begin{array}{c}
0 \\
0 \\
x_{i 1} x_{i 3}+b
\end{array}\right),
$$

where $B=\left(\begin{array}{ccc}0 & -1 & -1 \\ 1 & a & 0 \\ 0 & 0 & -c\end{array}\right), x_{i}=\left(\begin{array}{lll}x_{i 1} & x_{i 2} & x_{i 3}\end{array}\right)^{T}$. The following parameters have been used: $a=0.2, b=0.2, c=7$. 


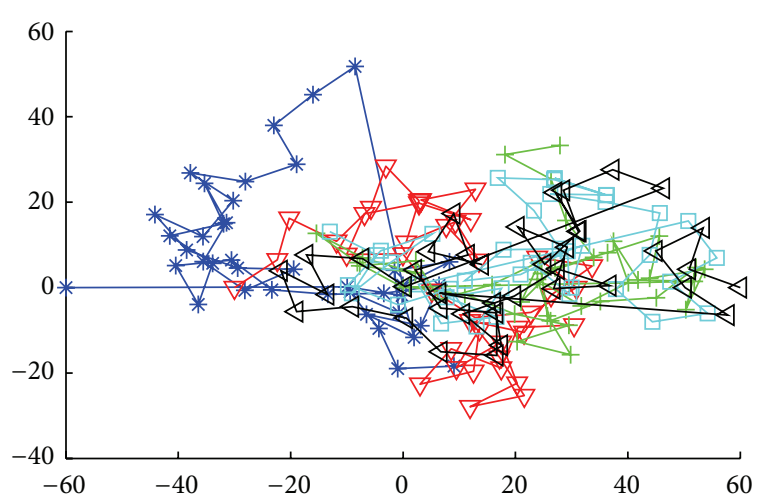

FIGURE 1: The position each agent during the movement.

Each agent node interacts at a given time with only those agents located within a neighborhood of an interaction radius. Here, we let periodic boundary conditions size $L=60$. The initial position $y_{i}(0)$ of agent $i$ in the plane is chosen at random. The initial orientation $\theta_{i}(0)=0$, other time units being chosen at each time unit with uniform probability in the interval $[0,2 \pi]$. Each agent is moving 40 time unit $(T=40)$. Then the position of each agent during the movement is shown in Figure 1.

When two agents interact (let interaction radius $R=30$ ), the state equations of each agent are changed to include diffusive coupling with the neighboring agent, acting on the state variable $x_{i 1}$. Based on these assumptions, the state dynamics of each agent can be described in terms of the following equations:

$$
\dot{x}_{i}=f\left(x_{i}\right)-\sigma \sum_{j=1}^{N} g_{i j}(t) E x_{j}+u_{i}
$$

where $i=1, \ldots, 5, f: R^{3} \rightarrow R^{3}$ is given by the Rössler dynamics, $E=\left[\begin{array}{lll}1 & 0 & 0 \\ 0 & 0 & 0 \\ 0 & 0 & 0\end{array}\right], g_{i j}(t)$ are the elements of a time-varying matrix $G(t)$, and $u_{i}=-d_{i} e_{i}, \dot{d}_{i}=e^{2 \lambda t}\left\|e_{i}\right\|^{2}$.

Obviously, one gets

$$
\bar{h}\left(x_{i}, s, t\right)=\left(\begin{array}{c}
0 \\
0 \\
x_{i 1} x_{i 3}-s_{1} s_{3}
\end{array}\right) .
$$

Similar to [23-25], since Rössler chaotic system has a chaotic attractor which is confined to abounded region $\phi \subset R^{n}$, there exists a constant $M$ satisfying $\left|x_{i j}\right|,\left|s_{j}\right| \leq M$ for $i=1,2, \ldots, N$ and $j=1,2,3$. Therefore,

$$
\begin{aligned}
\left\|\bar{h}\left(x_{i}, s, t\right)\right\| & =\sqrt{\left(x_{i 1} x_{i 3}-s_{1} s_{3}\right)^{2}} \\
& =\sqrt{\left(e_{i 1} x_{i 3}-s_{1} e_{i 3}\right)^{2}} \leq \sqrt{2} M\left\|e_{i}\right\| .
\end{aligned}
$$

$M$ can be got from the method similar to [24]. Thus, Assumption 7 holds.

Assume that $v_{i}(t)=100, \Delta t_{M}=0.1$ to guarantee the fastswitching condition Assumption 3. Thus, Assumptions 3 and

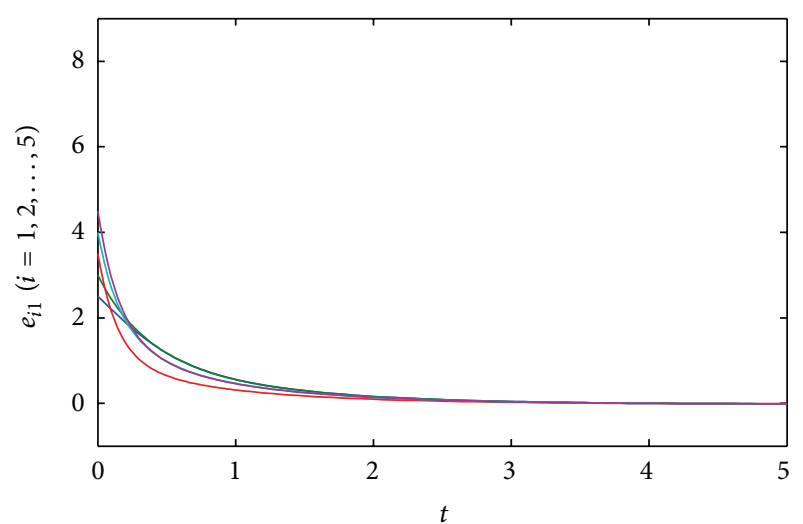

FIGURE 2: Synchronization errors of $e_{i 1}$ for the network.

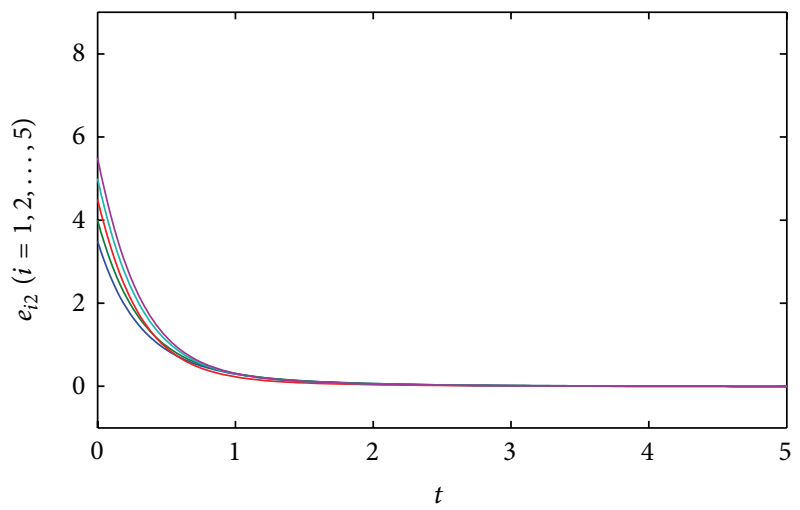

FIGURE 3: Synchronization errors of $e_{i 2}$ for the network.

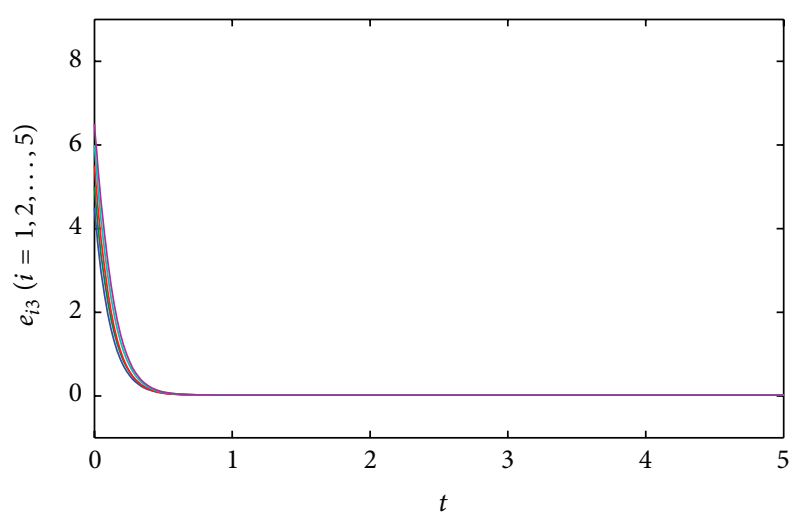

FIgURE 4: Synchronization errors of $e_{i 3}$ for the network.

7 hold. According to Theorem 8, the synchronous solution $s(t)$ of dynamical moving agent network (38) is globally exponentially stable. The other parameters are assigned as follows: $\lambda=1, d_{i}(0)=1, x_{i}(0)=(2+0.5 i, 3+0.5 i, 4+0.5 i)$, and $\sigma=1$. The synchronous error $e_{i}$ is shown in Figures 2,3 , and 4 . We learn from these figures that the synchronization errors can be globally exponentially stable for dynamical network (38). Therefore, we conclude that the states of the networks (38) can be globally exponentially synchronized with the state of each isolated Rössler system. 


\section{Conclusions}

Locally and globally adaptive exponential synchronization of moving agent network has been investigated in this paper. The network with decentralized controllers is considered as a large-scale nonlinear system with time-varying topological structure. An adequate Lyapunov function is constructed to deal with the problem of controlled synchronization so as to ensure the closed-loop system stability. Several network synchronization criteria for such network with time-varying topological have been obtained. And a numerical simulation of coupled Rössler system network is given, which demonstrates the effectiveness of the proposed synchronization scheme.

\section{Acknowledgments}

This research was supported in part by the Natural Science Foundation of Hebei under Grant no. F2012501030, Fundamental Research Funds for the Central Universities under Grant no. N100323012 from the Ministry of Education, and the National Natural Science Foundation of China under Grant no. 51105068.

\section{References}

[1] R. Albert and A.-L. Barabási, "Statistical mechanics of complex networks," Reviews of Modern Physics, vol. 74, no. 1, pp. 47-97, 2002.

[2] S. A. Pandit and R. E. Amritkar, "Characterization and control of small-world networks," Physical Review E, vol. 60, no. 2B, pp. R1119-R1122, 1999.

[3] S. H. Strogatz, "Exploring complex networks," Nature, vol. 410, no. 6825, pp. 268-276, 2001.

[4] M. Barahona and L. M. Pecora, "Synchronization in smallworld systems," Physical Review Letters, vol. 89, no. 5, pp. 054101/1-054101/4, 2002.

[5] X. F. Wang and G. Chen, "Synchronization in scale-free dynamical networks: robustness and fragility," IEEE Transactions on Circuits and Systems I, vol. 49, no. 1, pp. 54-62, 2002.

[6] S. Boccaletti, V. Latora, Y. Moreno, M. Chavez, and D.-U. Hwang, "Complex networks: structure and dynamics," Physics Reports, vol. 424, no. 4-5, pp. 175-308, 2006.

[7] X. Guo and J. Li, "A new synchronization algorithm for delayed complex dynamical networks via adaptive control approach," Communications in Nonlinear Science and Numerical Simulation, vol. 17, no. 11, pp. 4395-4403, 2012.

[8] W. Guo, "Lag synchronization of complex networks via pinning control," Nonlinear Analysis. Real World Applications, vol. 12, no. 5, pp. 2579-2585, 2011.

[9] S. Zheng, S. Wang, G. Dong, and Q. Bi, "Adaptive synchronization of two nonlinearly coupled complex dynamical networks with delayed coupling," Communications in Nonlinear Science and Numerical Simulation, vol. 17, no. 1, pp. 284-291, 2012.

[10] J. G. Barajas-Ramírez, "Robust synchronization of a class of uncertain complex networks via discontinuous control," Computers and Mathematics with Applications, vol. 64, no. 5, pp. 956-964, 2012.

[11] L. M. Pecora and T. L. Carroll, "Master stability functions for synchronized coupled systems," Physical Review Letters, vol. 80, no. 10, pp. 2109-2112, 1998.
[12] D. J. Stilwell, E. M. Bollt, and D. G. Roberson, "Sufficient conditions for fast switching synchronization in time-varying network topologies," SIAM Journal on Applied Dynamical Systems, vol. 5, no. 1, pp. 140-156, 2006.

[13] X.-B. Lu, B.-Z. Qin, and X.-Y. Lu, "Global synchronization of directed networks with fast switching topologies," Communications in Theoretical Physics, vol. 52, no. 6, pp. 1019-1924, 2009.

[14] B. Z. Qin and X. B. Lu, "Adaptive approach to global synchronization of directed networks with fast switching topologies," Physics Letters A, vol. 374, no. 38, pp. 3942-3950, 2010.

[15] M. Frasca, A. Buscarino, A. Rizzo, L. Fortuna, and S. Boccaletti, "Synchronization of moving chaotic agents," Physical Review Letters, vol. 100, no. 4, Article ID 044102, 2008.

[16] A. Buscarino, L. Fortuna, M. Frasca, and A. Rizzo, "Dynamical network interactions in distributed control of robots," Chaos, vol. 16, no. 1, Article ID 015116, 2006.

[17] S. Danø, P. G. Sørensen, and F. Hynne, "Sustained oscillations in living cells," Nature, vol. 402, no. 6759, pp. 320-322, 1999.

[18] L. Peng, Y. Zhao, B. Tian et al., "Consensus of self-driven agents with avoidance of collisions," Physical Review E, vol. 79, no. 2, Article ID 026113, 2009.

[19] M. Frasca, A. Buscarino, A. Rizzo, L. Fortuna, and S. Boccaletti, "Dynamical network model of infective mobile agents," Physical Review E, vol. 74, no. 3, Article ID 036110, 2006.

[20] L. Wang, H. Shi, and Y.-X. Sun, "Induced synchronization of a mobile agent network by phase locking," Physical Review E, vol. 82, no. 4, Article ID 046222, 2010.

[21] T. Liu, G. M. Dimirovski, and J. Zhao, "Exponential synchronization of complex delayed dynamical networks with general topology," Physica A, vol. 387, no. 2-3, pp. 643-652, 2008.

[22] J. Lu and D. W. C. Ho, "Local and global synchronization in general complex dynamical networks with delay coupling," Chaos, Solitons and Fractals, vol. 37, no. 5, pp. 1497-1510, 2008.

[23] K. Li and C. H. Lai, "Adaptive-impulsive synchronization of uncertain complex dynamical networks," Physics Letters A, vol. 372, no. 10, pp. 1601-1606, 2008.

[24] D. Li, J.-a. Lu, X. Wu, and G. Chen, "Estimating the ultimate bound and positively invariant set for the Lorenz system and a unified chaotic system," Journal of Mathematical Analysis and Applications, vol. 323, no. 2, pp. 844-853, 2006.

[25] C. Zhou and J. Kurths, "Dynamical weights and enhanced synchronization in adaptive complex networks," Physical Review Letters, vol. 96, no. 16, Article ID 164102, 2006. 


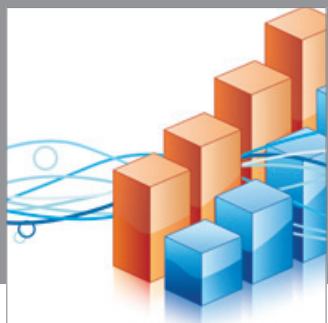

Advances in

Operations Research

mansans

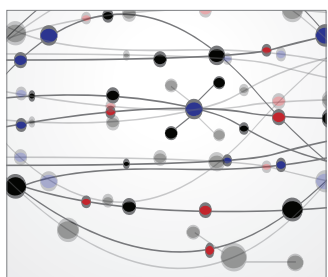

The Scientific World Journal
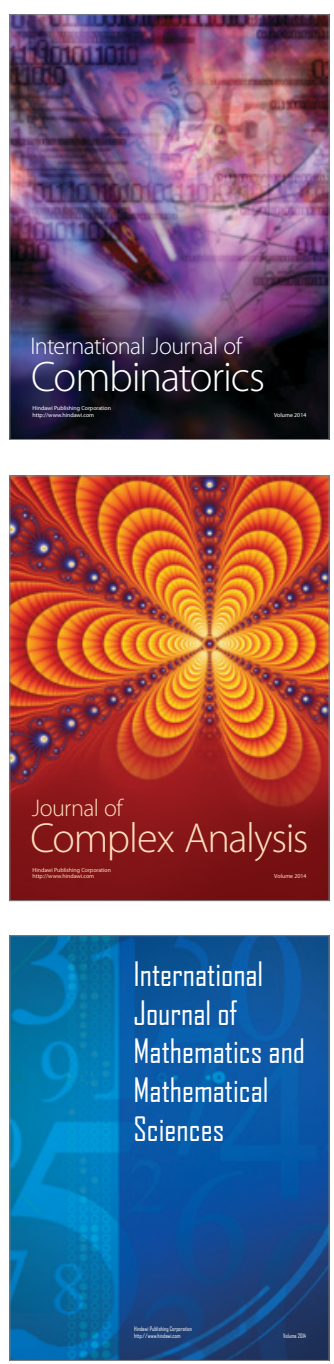
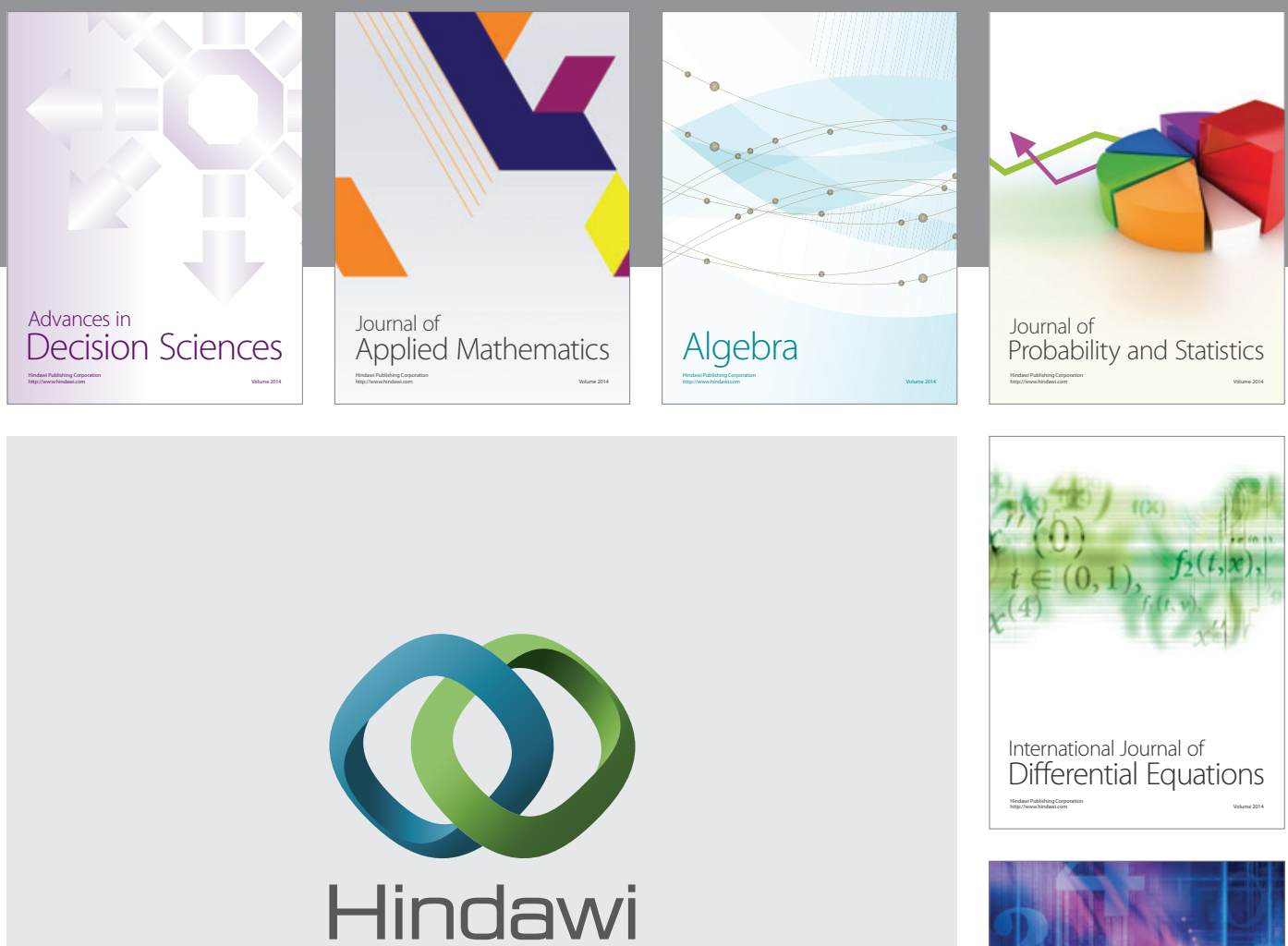

Submit your manuscripts at http://www.hindawi.com
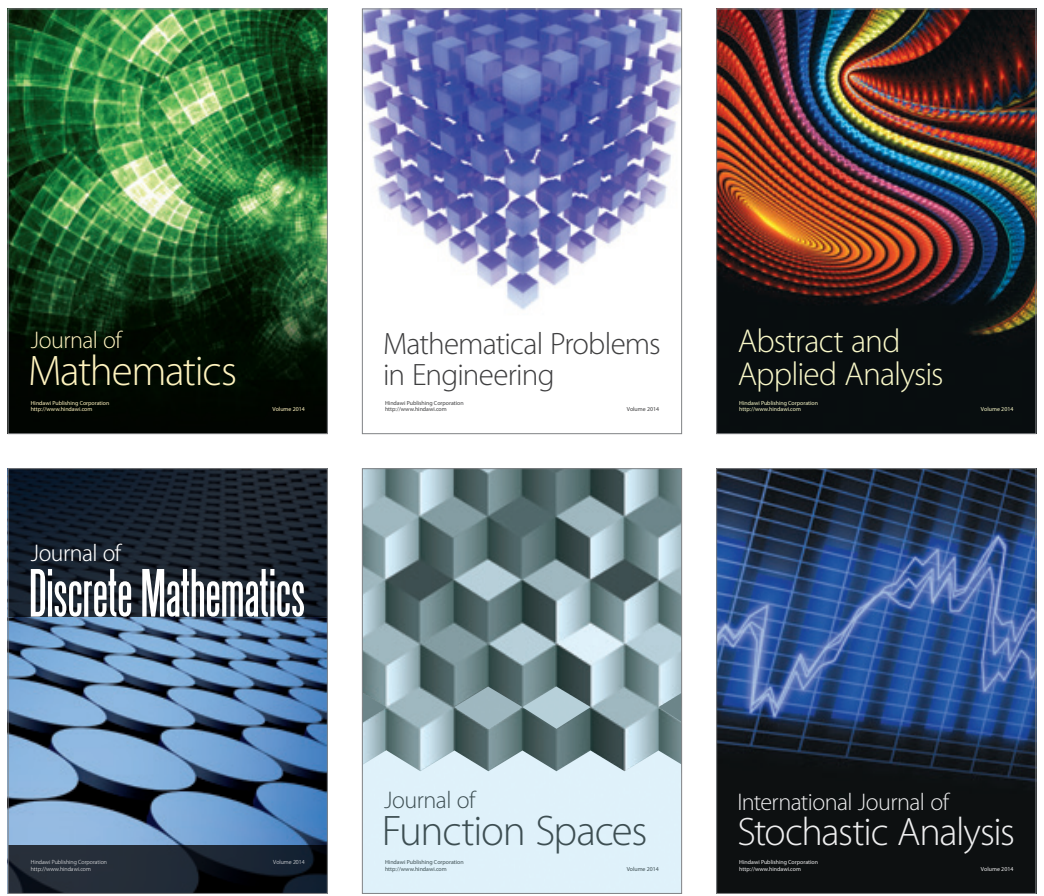

Journal of

Function Spaces

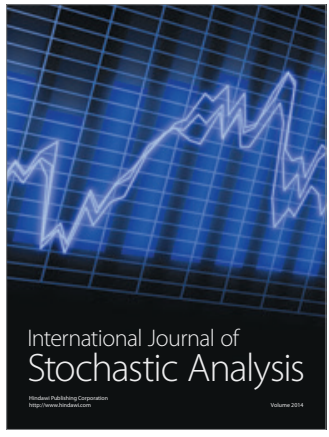

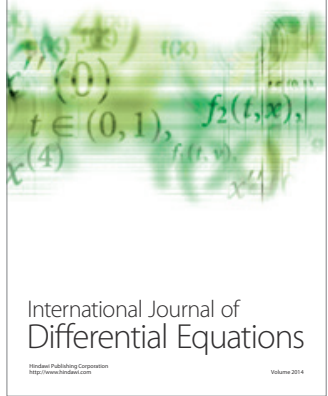
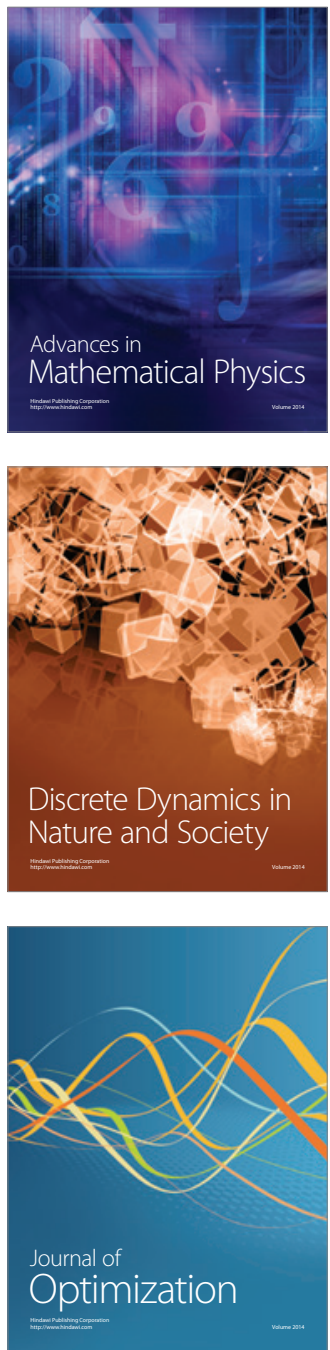